\title{
The Cutting Experiment of Full CNC Epicycloids Bevel Gear Cutting Machine
}

\author{
Meilin $\mathrm{Li}^{1, \mathrm{a}}$, Xiaolong Shen ${ }^{2, \mathrm{~b}}$, Yongxiang $\mathrm{Li}^{2}$ and Nanlin $\mathrm{Yu}^{2}$ \\ ${ }^{1}$ Hunan Urban Construction College, Xiangtan, 411101, China \\ ${ }^{2}$ Hunan Industry Polytechnic, Changsha, 410208, China \\ alimeilin0731@yeah.net, bShenXL64@163.com
}

Keywords: Motion Control, Epicycloids, Bevel Gears, Cutting Experiment.

\begin{abstract}
The relative motion relationship was derived among cutter, cradle, work gear and generating gear in the course of gear cutting based on the analysis the transmission line of mechanical epicycloids bevel gear cutting machine. Then the motions control general rules of epicycloids bevel gear cutting machine are concluded. Using those rules, a cutting simulation platform of epicycloids bevel gear is built in VERICUT. And a cutting experiment of epicycloids bevel gear is preformed in the YKD2275 full CNC bevel gear cutting machine.
\end{abstract}

\section{Introduction}

Commonly, five steps method is used for the spiral bevel gears are processing, and need to retract and index in the processing. And corresponding, the face hobbing method is use for the epicycloids bevel gears processing, the gear indexed continuously, and the rough cutting and finished cutting of both sides of the gear is completed in one step, so the processing efficiency is higher. On the other hand, the meshing line of this gear type is longer, and the transmission stability is better. The Gleason in American and Klingenberg in Germany have solved the face hobbing technology and have developed some CNC cutting machines [1-3]. However, the research on face hobbing method of epicycloids bevel gears is insufficient in domestic and has not yet developed the epicycloids bevel gear cutting machine tools.

Some research have been carried out in some universities such as China Agricultural University and Xi'an Jiaotong University on transmission structure of mechanical cutting machines, the principle of generating gear forming and the derivation of tooth surface [4-5]. But the research on the motion control of the full CNC epicycloids bevel gear cutting machine is shortage. To solve this problem, the kinematics law of epicycloids bevel gear cutting machine was studied in this paper, and machining simulation and cutting experiment was carried out to verify the kinematics law, which will play a good role in promoting the development of domestic epicycloids bevel gear cutting machine.

\section{The calculation of machine axes position for full CNC epicycloids bevel gear cutting machine}

The basic mathematical model for bevel gear cutting is shown in Figure 1, which is used to determine the parameters of the relative position between the generating gear and work gear. Put $\Sigma_{s}=\left\{O_{s}, \vec{i}_{s}, \vec{j}_{s}, \vec{k}_{s}\right\}$ as static coordinate system, $\Sigma=\{O, \vec{i}, \vec{j}, \vec{k}\}$ is a coordinate system to describe the course of cutting, and the origin $\mathrm{O}$ is on the axis of the cradle, coordinate plane $\vec{i} O \vec{j}$ perpendicular to the axis of the cradle, $\vec{i}$ is in the horizontal axial section of the cradle, $\vec{k}$ point to the outside of the cradle. $\Sigma_{c}=\left\{O_{c}, \vec{i}_{c}, \vec{j}_{c}, \vec{k}_{c}\right\}$ is a coordinate system to describe the cutter(generating gear), in which coordinate plane $\vec{i}_{c} O_{c} \vec{j}_{c}$ locates in the plane $\vec{i} o j$, and vector of $O_{c}$ in coordinate system $\Sigma$ is $\vec{S}$.Make $\Sigma_{o}=\left\{O_{o}, \vec{i}_{o}, \vec{j}_{o}, \vec{k}_{o}\right\}$ through the axial section of generating gear, in which point $O_{o}$ coincide with point $O_{c}, \vec{k}_{o}$ is on the axis of the generating gear, $\vec{j}_{o}$ point at $p$ from $O_{o}$. 


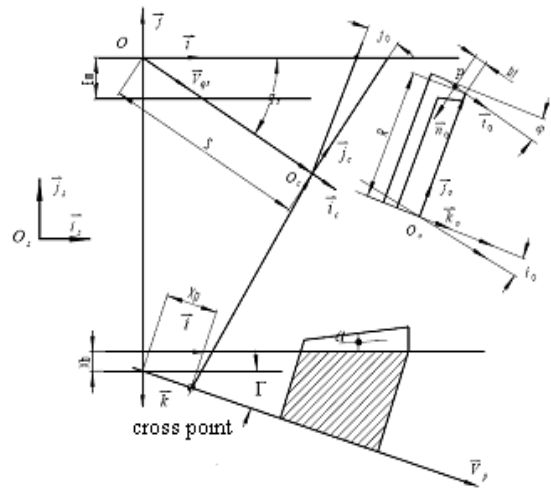

Fig1. The basic mathematical model for bevel cutting

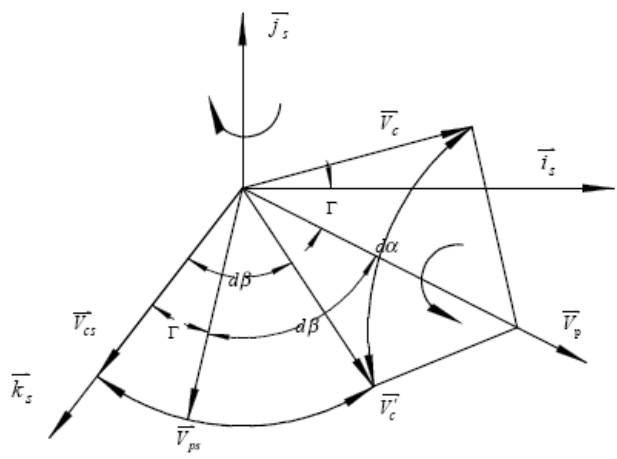

Fig2. The motion transformation diagram of full CNC machine

Then, the vector of cutter center from work gear cross point in the coordinate system $\Sigma$ can be expressed after adjusting gear and cutter position.

$$
\vec{V}_{l}=-\mathrm{X}_{\mathrm{p}} \vec{V}_{\mathrm{p}}-\mathrm{X}_{\mathrm{b}} \vec{k}+\mathrm{E}_{\mathrm{m}} \vec{j}+\mathrm{S} \vec{V}_{q t}
$$

In this formula, $\vec{V}_{p}=[\cos \Gamma, 0, \sin \Gamma]$ is a vector of gear axis, $\vec{V}_{q t}=\left[\cos q_{t},-\sin q_{t}, 0\right]$ is a vector of cutter position on cradle, $X_{p}$ is the machine center to back, $X_{b}$ is sliding base, $E_{m}$ is work offset, $S$ is radial distance of cutter, $\Gamma$ is machine root angle, $q_{t}$ is the angle between line from cutter center to cradle center and vector $\vec{i}$ in a moment [6].

Before the adjusting of cutter tilt angle, cutter axis vector $\vec{k}$ and coordinate axis of static coordinate system are in the same direction. After adjusting the cutter tilt body and cutter swivel body, cutter axis vector in the $\Sigma_{s}$ can be expressed as:

$$
\vec{V}_{c}=\mathrm{M}\left(q_{t}-j_{0}, \vec{k}\right) \mathrm{M}\left(i_{0}, \vec{i}\right) \vec{k}
$$

In this formula, $i_{0}$ is tilt angle. $j_{0}$ is swivel angle, $\mathrm{M}\left(i_{0}, \vec{i}\right)=\left[\begin{array}{ccc}1 & 0 & 0 \\ 0 & \cos \left(i_{0}\right) & -\sin \left(i_{0}\right) \\ \sin \left(i_{0}\right) & 0 & \cos \left(i_{0}\right)\end{array}\right]$ is a rotation matrix that a vector rotated an angle $i_{0}$ by $\vec{i}$.

However, the cutter axis vector in the full CNC cutting machine can not be changed. We must ensure the condition that the cutter axis vector direction always consistent with cradle axis $\overrightarrow{k_{s}}$ in the transforming process of the basic machine tool model into the full $\mathrm{CNC}$ machine model, and the relative motion between work gear and cutter should not be changed.

As Figure 2 shown, first let the entire machine to turn an angle $\alpha$ around work gear axis $\vec{V}_{\mathrm{p}}$, make the cutter axis vector $\vec{V}_{c}$ locate in the plane $\vec{i}_{s} o \vec{k}_{s}$. Then let the entire machine to rotate an angle $\beta$ around axis $\vec{j}_{s}$, make cutter axis vector $\vec{V}_{c}$ coincide with $\vec{k}_{s} 、 \alpha 、 \beta$ can be calculated by the two formula.

$$
\begin{aligned}
& \alpha=\operatorname{atan}\left(\frac{\vec{V}_{c} \cdot \vec{j}}{\vec{V}_{c} \cdot\left(\vec{V}_{p} \times \vec{j}\right)}\right) \\
& \left.\begin{array}{l}
\beta=\pi / 2-\varphi-\Gamma \\
\varphi=a \cos \left(\vec{V}_{c} \cdot \vec{V}_{p}\right)
\end{array}\right\}
\end{aligned}
$$

Therefore, The cutter axis vector $\vec{V}_{c}$ in the static coordinate $\Sigma_{s}$ can be expressed as:

$$
\overrightarrow{V_{c s}}=\mathrm{M}\left(\alpha, \vec{V}_{p}\right) \mathrm{M}(\beta, \vec{j}) \vec{V}_{c}=\vec{k}_{s}
$$

Relatively, the vector $\vec{V}_{l}$ which means the relative position between work gear and the cutter center in the static coordinate $\Sigma_{s}$ after transformation can be expressed as: 


$$
\vec{V}_{l s}=\mathrm{M}\left(\alpha, \vec{V}_{p}\right) \mathrm{M}(\beta, \vec{j}) \vec{V}_{l}
$$

Because the cutter speed is constant in the processing, $C=\theta$ can be set. $\theta$ is cutter rotation angle. Based on the analysis of above, the rotation angle of work gear used for indexing is:

$$
A_{1}=\left[-\theta+\left(q_{t}-q_{s}\right)\right] z_{0} / z_{i}
$$

The rotation angle of work gear used for generating movement is:

$$
A_{2}=R_{a q}\left(q_{t}-q_{s}\right)-\alpha
$$

In which, $z_{0}$ is the groups of the cutter blades, $z_{i}$ is the teeth number of gear. If let crossing point of gear as the origin of $\mathrm{CNC}$ machine, the cutter pathway of gear cutting processing can be calculated by used formula:

$$
\left.\begin{array}{l}
X=\vec{V}_{l s} \cdot \vec{i}_{s} \\
Y=\vec{V}_{l s} \cdot \vec{j}_{s} \\
Z=\vec{V}_{l s} \cdot \vec{k}_{s} \\
B=\Gamma+\beta \\
\begin{array}{l}
C \\
A
\end{array}=\theta \\
A A_{1}+A_{2}
\end{array}\right\}
$$

$R_{a q}$ is ratio of roll; $q_{s}$ is start angle of cradle in processing.

\section{Machining Simulation and Gear Cutting Experiment}

In order to verify the above derivation, a processing simulation platform of $\mathrm{CNC}$ epicycloids bevel gear cutting machine is build in VERICUT software. As Figure 3(a) shown, the column of machine can be moved along the axis $\mathrm{X}$ on the bed, the skateboard on the column can be moved along the axis $\mathrm{Y}$. The cutter is installed on the skateboard and turn around with axis $\mathrm{C}$. The linkages between axis $\mathrm{X}$ and axis $\mathrm{Y}$ drive the cutter movement according to the arc path, and simulate the rotation of the cradle in the course of cutting. The sliding base can be moved along the axis $\mathrm{Z}$ to control cutting depth of the tooth. The axis B is placed on sliding base for adjust the root angle. Workpiece box is installed on the axis $\mathrm{B}$, when the axis B placed at zero degree, the direction of work gear axis A is parallel to the axis $\mathrm{X}$. The components tree of the simulation machine is shown in Figure 3(b). An experiment is carried out. The basic parameters of work gear is shown in Table 1.Using the above algorithm to generate the tool path, rewrite it to NC program and import to the simulation platform, then perform cutting simulation after simplified the cutter.

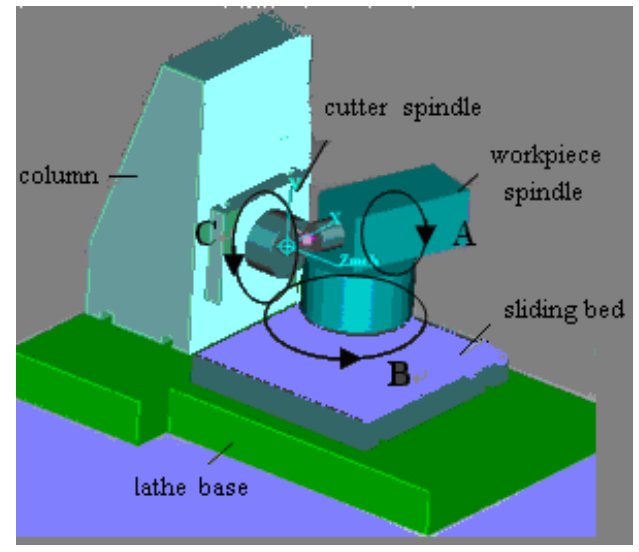

(a)

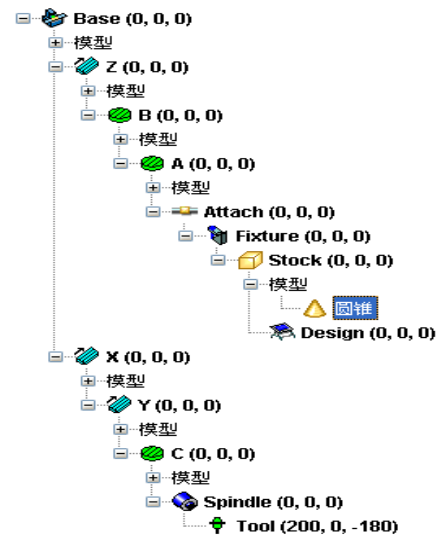

(b)

Fig 3. The gear cutting simulation platform

The result of simulation is shown in Figure 4. We can see that the tooth indexing and tooth form is normal. Then import the NC program into the YKD2275 full CNC bevel gear cutting machine which manufactured by Shanghai No.1 Machine Tool Works. The nylon gear blank is used in cutting 
experiment. The process of gear cutting is shown in Figure 5, and the gear after processing as shown in Figure 6. We can see that tooth taper is satisfactory, the cutter interference doesn't appear at tooth root, the tooth surface is continuous and the tooth form is consistent with the theoretical result.

Table1. Basic parameters of gear sets

\begin{tabular}{ccc}
\hline & Pinion & Gear \\
\hline Tooth Number & 9 & 40 \\
\hline Module & \multicolumn{2}{c}{11.400} \\
\hline Sharft Angle & \multicolumn{2}{c}{$90^{\circ}$} \\
\hline Offset & \multicolumn{2}{c}{44.45} \\
\hline Mean spiral angle & $46^{\circ} 30^{\prime}$ & $33^{\circ} 45^{\prime}$ \\
\hline Face width & 76.74 & 70.00 \\
\hline Hand of spiral & LH & RH \\
\hline Average pressure angle & $22^{\circ}$ \\
\hline
\end{tabular}

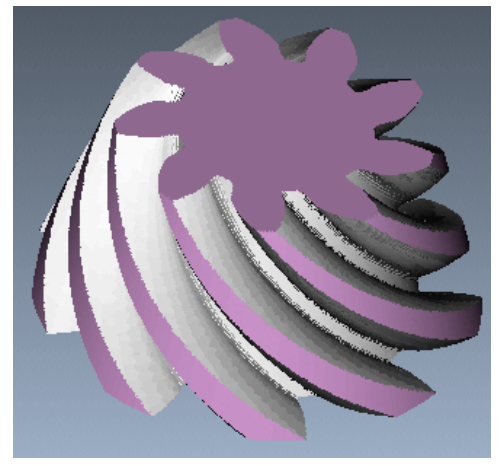

Fig 4. Result of cutting simulation

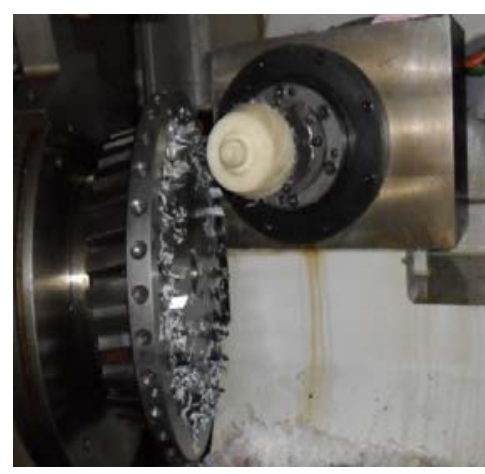

Fig 5. Process of cutting experiment

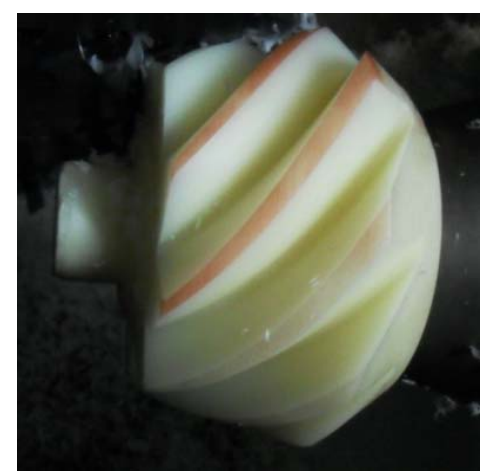

Fig 6.The gear after face hobbing

\section{Summary}

In this paper, the drive line of mechanical epicycloids bevel gear cutting machine is analyzed. The relative motion is derived among cutter, cradle, generating gear and work gear. The axes positions calculation method of machine cutting is researched, then a general law of motion control of full CNC epicycloids bevel gear cutting machine is summed up. At last those methods and calculation is verified by cutting simulation and experiment. This study has a good practical value for understanding the forming principle of epicycloids bevel gear deeply and the development of domestic full CNC epicycloids bevel gear cutting machine.

\section{Acknowledgements}

This project is supported by Science and Technology Fund of Hunan Provincial Science and Technology Department (No.2011GK3090).

\section{References}

[1] Qi Fan, Kinematical Simulation of Face Hobbing Indexing and Tooth Surface Generation of Spiral Bevel and Hypoid Gears, Gear Technology, 2006(1), p. 30-38.

[2] Yi-Pei Shih, Zhang-Hua Fong, Mathematical Model for a Universal Face Hobbing Hypoid Gear Generator. ASME Journal of Mechanical Design, 129(2007), p. 38-47.

[3] J.F.G. Oliveira, T.V. Franca and J.P. Wang: CIRP Ann. Vol. 57 (2008), p. 329-332.

[4] Fang Chen. Yunfei Zhou. Xiaoqing Li. CNC processing sports transform analysis for gear of Oerlikon. Machinery and Electronics. 2002(1), p. 73-75.

[5] X.L. Shen, Y.X. Luo, L.X. Zhang and H. Long: Adv. Mater. Res. Vol. 156-157(2011).

[6] G.L. Samuel and M.S. Shunmugam: Precision Eng. Vol. 24 (2000), p. 251-263. 